\title{
Effects of a High Protein/Low Carbohydrate Versus a Standard Hypocaloric Diet on Weight and Cardiovascular Risk Factors, Role of a Lys656asn Polymorphism of Leptin Receptor Gene
}

\author{
Daniel de Luis ${ }^{1,2 *}$, Rocío Aller ${ }^{1}$, Olatz Izaola ${ }^{1,2}$, Primo $D^{1}$ and Romero $E^{1,2}$
}

${ }^{1}$ Center of Investigation of Endocrinology and Nutrition, Medicine School, Spain

${ }^{2}$ Department of Endocrinology and Nutrition, Hospital Clínico Universitario, Spain

\begin{abstract}
Background: The SNP of the leptin receptor (Lys656Asn) has been related with metabolic parameters. The aim of our design was to evaluate the influence of Lys656Asn variant of Leptin receptor gene on weight loss and metabolic parameters secondary to a high protein/low carbohydrate vs. a standard hypocaloric diets.

Design: A total of 280 obese subjects were randomly allocated to one of two diets during 9 months (Diet HP (high protein-low carbohydrate hypocaloric diet) and Diet S (standard protein hypocaloric diet)).

Results: After both diets and in both genotypes, body mass index (BMI), fat mass, weight, systolic blood pressure and waist circumference decreased, without differences between both diets. With the diet type HP and in both genotypes, triglycerides decreased. In subjects with Lys656Lys genotype, glucose, insulin levels, HOMA-R, total cholesterol and LDL- total cholesterol decreased after HP diet. No statistical changes were detected in Asn allele carriers. After diet $S$ and in patients with Lys656Lys genotypes, only total cholesterol, triglycerides and LDL- total cholesterol decreased, without changes in Asn allele carriers.
\end{abstract}

Conclusion: Obese subjects with Asn656 allele have a different metabolic response than Lys656Lys genotype subjects, secondary to the same weight loss with two different hypocaloric diets.

Keywords: High protein/low carbohydrate; Standard hypocaloric diet; LYS656ASN; LEPR; Leptin

Abbreviations: BMI: Body Mass Index; HOMA: Homeostasis Model Assessment; IR: Insulin Resistance; LEPR: Leptin Receptor; WC: Waist Circumference; FFM: Fat Free Mass; FM: Fat Mass; WHR: Waist to Hip Ratio; SBP: Systolic Blood Pressure; DBP: Diastolic Blood Pressure

\section{Introduction}

The incidence of obesity is increasing all Western Countries. This dramatically increasing in obesity prevalence has led to an important increase of investigation on adipose tissue and its role in metabolism, inflammation and other physiologic processes [1]. Obesity has a low grade systemic inflammation and adipose tissue is considered an active secretory organ of a new family of molecules called adipokines (for example, leptin). Leptin is a protein containing 167 amino acids, demonstrates structural similarities with cytokines and is mainly produced by white adipose tissue. Some studies have confirmed that other tissues express leptin, including ovaries, placenta, stomach, pituitary, skeletal muscle and liver [2]. This adipokine acts as an afferent satiety signal, regulating appetite in humans. Obese patients may be leptin resistant and are characterized by high, rather than low, levels of leptin [3]. Considering everything previously mentioned, the role of leptin in the obesity is an area of important investigation.

Some genetic variant (polymorphisms) in leptin receptor gene have been studied with contradictory results [4]. One of them is the SNP on codon 656, this change produce a modification in electric charge with a possibility to be functional. Patients with rs8179183 variant of LEPR gene have a different metabolic response than wild type after weight loss secondary to different diets [5-7]. In these previous studies, only leptin levels had a significant decrease in wild group. However, although dietary intakes are the main tool to decrease weight in obese subjects, responded to diet was heterogeneous, and a role for genetic turned determinants in the inter individual variation is postulated [8].
A recent meta-analysis with low-carbohydrate/high protein diets has shown that such diets have benefit effects on weight loss, metabolic parameters and cardiovascular risk factors [9]. As far as we know, no interventional designs have been realized in order to evaluate the effect of this polymorphism on response to diets of this type.

The aim of our study was to evaluate the effect of this polymorphism on metabolic parameters and weight loss secondary to a high protein/ low carbohydrate vs. a standard hypocaloric diets (1000 kcal/day) during 9 months.

\section{Methods and Subjects}

\section{Subjects}

A sample of 280 obesity non diabetic outpatients was analyzed in a prospective way. Written informed consent was fulfilled from all subjects. Exclusion criteria included history of stroke or cardiovascular disease during the previous 12 months, total cholesterol $>275 \mathrm{mg} / \mathrm{dl}$, triglycerides $>350 \mathrm{mg} / \mathrm{dl}$, fasting plasma glucose $>110 \mathrm{mg} / \mathrm{dl}$, blood pressure $>140 / 90 \mathrm{mmHg}$, as well as the use of treatments to diabetes mellitus (insulin, sulphonilurea, dypeptidil type IV inhibitors drugs, thiazolidinedions), glucocorticoids, antineoplasic agents, angiotensin

*Corresponding author: Dr. Daniel de Luis, Professor of Endocrinology and Nutrition, Executive Director of Center of Investigation of Endocrinology and Clinical Nutrition, Medicine School, Valladolid University, Valladolid, Spain, Tel: +34983423000; E-mail: dadluis@yahoo.es

Received May 27, 2016; Accepted June 17, 2016; Published June 24, 2016 Citation: de Luis D, Aller R, Izaola O, Primo D, Romero E (2016) Effects of a High Protein/Low Carbohydrate Versus a Standard Hypocaloric Diet on Weight and Cardiovascular Risk Factors, Role of a Lys656asn Polymorphism of Leptin Receptor Gene. J Metabolic Synd 5: 206. doi:10.4172/2167-0943.1000206

Copyright: $\odot 2016$ de Luis D, et al. This is an open-access article distributed under the terms of the Creative Commons Attribution License, which permits unrestricted use, distribution, and reproduction in any medium, provided the original author and source are credited. 
Citation: de Luis D, Aller R, Izaola O, Primo D, Romero E (2016) Effects of a High Protein/Low Carbohydrate Versus a Standard Hypocaloric Diet on Weight and Cardiovascular Risk Factors, Role of a Lys656asn Polymorphism of Leptin Receptor Gene. J Metabolic Synd 5: 206. doi:10.4172/2167-0943.1000206

Page 2 of 5

receptor blockers, angiotensin converting enzyme inhibitors, psychoactive medications, statins and other lipid drugs. This study was conducted according to the guidelines laid down in the Declaration of Helsinki and all procedures involving human subjects were approved by the Local Ethical Committee.

\section{Procedure}

Basal fasting glucose, total cholesterol, c-reactive protein (CRP), insulin, insulin resistance (HOMA-IR), LDL-cholesterol, HDLcholesterol, plasma triglycerides concentration and adipokines (leptin, resistin, adiponectin) levels were determined within the start of the trial and repeated after 3 months and 9 months of both dietary intervention. Weight, height, and blood pressure measures were determined within the start of the trail and repeated 3 and 9 months of intervention. A tetrapolar bioimpedance was realized in order to measure fat mass. These measures were realized at same time of the day (morning). Genotype of Lys656Asn variant of Leptin receptor gene was studied.

280 obese patients were randomly allocated to one of two diets. Diet HP (a hypocaloric diet, high protein-low carbohydrate) consisted in a diet of $1050 \mathrm{cal} /$ day, $33 \%$ of fats $(39.0 \mathrm{~g} /$ day), $33 \%$ of carbohydrates $(86.1 \mathrm{~g} /$ day) and $34 \%$ of proteins $(88.6 \mathrm{~g} /$ day $)$. The distribution of fats was; $63.8 \%$ of monounsaturated fats, $23.5 \%$ of saturated fats and $12.6 \%$ of polyunsaturated fats. Diet $S$ (hypocaloric diet, standard protein) consisted in a diet of $1093 \mathrm{cal} /$ day, $27 \%$ fats $(32.6 \mathrm{~g}), 53 \%$ carbohydrates $(144.3 \mathrm{~g} /$ day $)$ and $20 \%$ proteins $(55.6 \mathrm{~g} /$ day). The distribution of fats was; $67.4 \%$ of monounsaturated fats, $20.9 \%$ of saturated fats, and $11.6 \%$ of polyunsaturated fats. The exercise program was an aerobic physical activity at least 3 times per week ( $60 \mathrm{~min}$ each). The adherence of these diets was recorded each 7 days with a phone call in order to improve both diets. National composition food tables were used as reference [10].

\section{Genotyping of LEPR gene polymorphism}

Probes and oligonucleotide primers were designed with the Beacon Designer 4.0 (Premier Biosoft International ${ }^{\circ}$, LA, CA). The polymerase chain reaction (PCR) was realized with $250 \mathrm{ng}$ of genomic DNA, $0.5 \mu \mathrm{L}$ of each primer (primer forward: 5'-GCA GTT CCT ATG AGA GGA CC-3'; primer reverse: 5'-AAA TTG GGA ATA CCT TCC AAA GT-3'), and $0.25 \mathrm{uL}$ of each probes wild probe: (5'-Fam-AGT GAC ATT TTT CTC CTT TTT CAT AGT ATC-Tamra-3' and mutant probe: 5'-HexAGT GAC ATT TTT CTC GTT TTT CAT AGT AT- Tamra - $3^{\prime}$ ) in a $25 \mu \mathrm{L}$ final volume (Termociclador iCycler IQ (Bio-Rad $\left.{ }^{\star}\right)$, Hercules, CA). DNA was denaturated at $95^{\circ} \mathrm{C}$ for $3 \mathrm{~min}$; this was followed by 50 cycles of new denaturation at $95^{\circ} \mathrm{C}$ for $15 \mathrm{~s}$, and annealing at $59.3^{\circ} \mathrm{C}$ for $45 \mathrm{~s}$ ). The PCR were run in a $25 \mu \mathrm{L}$ final volume containing $12.5 \mu \mathrm{L}$ of IQTM Supermix (Bio-Rad ${ }^{\oplus}$, Hercules, CA) with hot start Taq DNA polymerase. The Hardy Weinberg equilibrium was examined with $\mathrm{p}$ value $>0.05$.

\section{Anthropometric measurements and blood pressure}

Weight was measured to an accuracy of $0.1 \mathrm{Kg}$ and BMI was calculated as body weight/(height ${ }^{2}$ ). Hip (widest diameter over greater trochanters) and waist (narrowest diameter between xiphoid process and iliac crest) circumferences to derive waist-to hip ratio (WHR) were determined, too. Body electrical bio impedance was used to determine body composition [11] (Biodynamics Model 310e, Seattle, WA, USA).

Blood pressure was measured twice after a 10 minutes rest with a random zero mercury sphygmomanometer and averaged.

\section{Biochemical assays}

Cholesterol and triglyceride concentrations were measured by enzymatic colorimetric assay (Technicon Instruments, Ltd., New York, N.Y., USA), while HDL cholesterol was measured enzymatically in the supernatant after precipitation of other lipoproteins with dextran sulphate-magnesium. Low-density lipoprotein cholesterol was determined using Friedewald formula.

Plasma glucose levels were determined by using an automated glucose oxidase method (Glucose analyser 2, Beckman Instruments, Fullerton, California). Insulin was determined by enzymatic colorimetric (Insulin, WAKO Pure-Chemical Industries, Osaka, Japan) [12] and the homeostasis model assessment for insulin resistance (HOMA-IR) was determined using these values [13]. CRP was determined by immunoturbimetry (Roche Diagnostics $\mathrm{GmbH}$, Mannheim, Germany), with a normal range of (0-7 mg/dl) and analytical sensivity $0.5 \mathrm{mg} / \mathrm{dl}$.

Plasma hormone levels were measured using the multiplex Biorad $\odot 10$ plex assay following manufacturer's instructions (Bio$\mathrm{Rad}^{\circ}$, Hercules, CA). This system lets for quantitative measurement of different molecules, while consuming a small amount of material; resistin, leptin and adiponectin. Limits of detection were as follows (pg/ $\mathrm{ml})$ : resistin (1.4), leptin (1.8), and adiponectin (3.8).

\section{Statistical analysis}

Sample size was calculated to observed differences over $10 \%$ in leptin levels with $90 \%$ power and $5 \%$ significance $(n=130)$. The distribution of variables was analyzed with Kolmogorov-Smirnov test. Quantitative variables with normal distribution were analyzed with a two-tailed, paired Student's-t test. A Chi square test was used to evaluate the Hardy-Weinberg equilibrium. Non-parametric parameters were analyzed with the Wilcoxon test. A mixed model has been used within and between subjects to test the interaction between the polymorphism groups and outcome variables, due to the repeated measures study design. Qualitative parameters were analyzed with the chi-square test, with Yates correction as necessary, and Fisher's test. The statistical analysis was realized for the combined Lys656/Asn656 and Asn656/Asn656 as a group and genotype Lys656/Lys 656 as other group (dominant model). A p-value under 0.05 was considered statistically significant.

\section{Results}

280 patients gave signed consent and were included in the study. The mean age was $49.5 \pm 11.2$ years and the mean body mass index 37.8 \pm 3.2 , with 75 males $(26.8 \%)$ and 205 females (73.2\%). One hundred and eighty eight patients (54 males/134 females) (67.1\%) had the genotype Lys656/Lys656 and 92 (32.9\%) patients (21 males/71 females) Lys656/Asn656 ( $n=81,28.9 \%)$ or Asn656/Asn656 ( $n=11,3.9 \%)$ (Asn allele carriers). The Hardy Weinberg equilibrium was fulfilled $\mathrm{p}=0.23$. Sex frequency was equal in both groups, males $(22.7 \%$ vs $23.6 \%)$ and females $(77.3 \%$ vs $76.4 \%$ ). Age was equal in both groups (Lys656Lys genotype: $49.7 \pm 10.2$ years vs Asn carriers group: $48.9 \pm 12.7$ years:ns)

In the 139 subjects (94 Lys656Lys genotype and 45 Asn allele carriers) randomized to diet HP, basal determination of dietary intake with a 3 days written food register detected a basal calorie intake of $2099.8 \pm 432.6 \mathrm{kcal} /$ day, a fat intake of $90.0 \pm 29.3 \mathrm{~g} /$ day $(41.1 \%$ of calories), a carbohydrate intake of $219.38 \pm 30.9 \mathrm{~g} /$ day $(40.8 \%$ of calories) and a protein intake of $81.2 \pm 59.1 \mathrm{~g} /$ day $(27.1 \%$ of calories). During the trial, these patients attained the recommendations of diet; $1010.4 \pm 91.1$ calories $(34.2 \%$ of carbohydrates, $32.6 \%$ of lipids and 
Citation: de Luis D, Aller R, Izaola O, Primo D, Romero E (2016) Effects of a High Protein/Low Carbohydrate Versus a Standard Hypocaloric Diet on Weight and Cardiovascular Risk Factors, Role of a Lys656asn Polymorphism of Leptin Receptor Gene. J Metabolic Synd 5: 206. doi:10.4172/2167-0943.1000206

Page 3 of 5

$36.2 \%$ of proteins). The 141 subjects (94 Lys656Lys genotype and 47 Asn allele carriers) randomized to diet $\mathrm{S}$, basal assessment of dietary intake with a 3 days written food register showed a basal calorie intake a calorie intake of $2028.4 \pm 394.1 \mathrm{kcal} /$ day, a fat intake of $91.0 \pm 13.1$ $\mathrm{g} /$ day (38.4\% of calories), a carbohydrate intake of $213.9 \pm 28.3 \mathrm{~g} / \mathrm{day}$ ( $42.7 \%$ of calories), and a protein intake of $90.9 \pm 11.9 \mathrm{~g} /$ day $(19.9 \%$ of calories). During the trial, these patients reached the recommendations of diet; $1008.8 \pm 97.1$ calories $(51.8 \%$ of carbohydrates, $29.4 \%$ of lipids and $18.8 \%$ of proteins).

Anthropometric parameters of patients at baseline and at 3-9 months of intervention are shown in Table 1. With the diet type HP (high protein hypocaloric diet) and in both genotype groups (Lys656Lys vs Lys656Asn+Asn656Asn), body mass index (BMI) $\left(-2.2 \pm 1.1 \mathrm{~kg} / \mathrm{m}^{2}\right.$ vs $\left.-1.9 \pm 1.3 \mathrm{~kg} / \mathrm{m}^{2}: \mathrm{ns}\right)$, weight $(-6.1 \pm 2.0 \mathrm{~kg}$ vs $-6.7 \pm 4.0 \mathrm{~kg}: \mathrm{ns})$, fat mass $(-5.2 \pm 3.1 \mathrm{~kg}$ vs $-5.1 \pm 3.0 \mathrm{~kg}: \mathrm{ns})$, systolic blood pressure $(-4.1$ $\pm 2.0 \mathrm{mmHg}$ vs $-3.1 \pm 1.9 \mathrm{mmHg}: \mathrm{ns})$ and waist circumference $(-6.1$ $\pm 5.0 \mathrm{~cm}$ vs $-6.3 \pm 4.5 \mathrm{~cm}$ : ns) decreased. There were not significant differences between the changes (on weight, waist circumference, BMI, systolic blood pressure and fat mass) in either genotype group. With the diet type $\mathrm{S}$ (Standard hypocaloric diet) and in both groups, body mass index $\left(-1.3 \pm 1.0 \mathrm{~kg} / \mathrm{m}^{2}\right.$ vs $\left.-1.1 \pm 0.9 \mathrm{~kg} / \mathrm{m}^{2}: \mathrm{ns}\right)$, weight $(-4.8 \pm$ $3.0 \mathrm{~kg}$ vs $-5.1 \pm 3.2 \mathrm{~kg} \mathrm{~ns})$, fat mass $(-4.3 \pm 3.1 \mathrm{~kg}$ vs $-4.0 \pm 3.0 \mathrm{~kg}: \mathrm{ns})$, blood pressure $(-4.0 \pm 1.9 \mathrm{mmHg}$ vs $-4.4 \pm 1.9 \mathrm{mmHg}: \mathrm{ns})$ and waist circumference $(-5.2 \pm 4.0 \mathrm{~cm}$ vs $-6.0 \pm 3.9 \mathrm{~cm}$ : $\mathrm{ns})$ decreased. There were no statistical differences between the effects in either genotype group with diet $S$. Table 2 shows the metabolic parameters. With the diet type HP and in both genotypes, triglycerides $(-19.0 \pm 8.1 \mathrm{mg} / \mathrm{dl}$ vs $-13.1 \pm 7.9 \mathrm{mg} / \mathrm{dl} ; \mathrm{ns})$ decreased. Only in patients with Lys656Lys genotype, glucose $(-4.1 \pm 1.1 \mathrm{mg} / \mathrm{dl}$ vs $-1.1 \pm 2.5 \mathrm{mg} / \mathrm{dl}$; $\mathrm{p}<0.05)$, insulin levels $(-2.6 \pm 2.0 \mathrm{UI} / \mathrm{L}$ vs $-0.9 \pm 2.0 \mathrm{UI} / \mathrm{L} ; \mathrm{p}<0.05)$, HOMA-IR $(-0.5 \pm 1.0$ units vs $-0.1 \pm 1.1$ units; $\mathrm{p}<0.05)$, total cholesterol $(-12.0 \pm 8.1 \mathrm{mg} / \mathrm{dl}$ vs $-5.1 \pm 6.1 \mathrm{mg} / \mathrm{dl} ; \mathrm{p}<0.05)$, and LDL- total cholesterol $(-6.8 \pm 4.1 \mathrm{mg} / \mathrm{dl}$ vs $-2.9 \pm 3.1 \mathrm{mg} / \mathrm{dl} ; \mathrm{p}<0.05)$ decreased. All these parameters remained

\begin{tabular}{|c|c|c|c|c|c|c|c|c|c|c|c|c|c|c|c|c|}
\hline \multirow[t]{3}{*}{ Characteristics } & \multicolumn{8}{|c|}{ DIET HP (n=139) } & \multicolumn{8}{|c|}{ DIET S $(n=141)$} \\
\hline & \multicolumn{4}{|c|}{ LysLys(n=94) } & \multicolumn{4}{|c|}{ LysAsn+ AsnAsn ( $n=45)$} & \multicolumn{4}{|c|}{ LysLys (n=94) } & \multicolumn{4}{|c|}{ LysAsn+ AsnAsn ( $n=47)$} \\
\hline & 0 time & $\begin{array}{c}\text { At } 3 \\
\text { months }\end{array}$ & $\begin{array}{c}\text { At } 9 \\
\text { months }\end{array}$ & $\begin{array}{c}\mathbf{p} \\
\text { value }\end{array}$ & 0 time & $\begin{array}{c}\text { At } 3 \\
\text { months }\end{array}$ & $\begin{array}{c}\text { At } 9 \\
\text { months }\end{array}$ & $\begin{array}{c}p \\
\text { value }\end{array}$ & 0 time & $\begin{array}{c}\text { At } 3 \\
\text { months }\end{array}$ & $\begin{array}{c}\text { At } 9 \\
\text { months }\end{array}$ & p value & 0 time & $\begin{array}{c}\text { At } 3 \\
\text { months }\end{array}$ & $\begin{array}{c}\text { At } 9 \\
\text { months }\end{array}$ & p value \\
\hline BMI & $\begin{array}{c}34.7 \pm \\
5.3\end{array}$ & $\begin{array}{c}33.0 \pm \\
4.0^{*}\end{array}$ & $\begin{array}{c}32.4 \pm \\
4.0^{*}\end{array}$ & 0.01 & $\begin{array}{c}34.9 \pm \\
6.0\end{array}$ & $\begin{array}{c}33.2 \pm \\
6.0^{*}\end{array}$ & $\begin{array}{c}33.0 \pm \\
5.1^{*}\end{array}$ & 0.01 & $\begin{array}{c}34.6 \pm \\
5.9\end{array}$ & $\begin{array}{c}34.1 \pm \\
4.2^{*}\end{array}$ & $\begin{array}{c}33.9 \pm \\
5.0 *\end{array}$ & 0.01 & $\begin{array}{c}35.1 \pm \\
4.1\end{array}$ & $\begin{array}{c}34.2 \pm \\
5.0^{*}\end{array}$ & $\begin{array}{c}34.0 \pm \\
5.1^{*}\end{array}$ & 0.02 \\
\hline Weight (kg) & $\begin{array}{c}91.7 \pm \\
14.6\end{array}$ & $\begin{array}{l}87.5 \pm \\
13.1^{*}\end{array}$ & $\begin{array}{c}85.6 \pm \\
10.1^{*}\end{array}$ & 0.02 & $\begin{array}{c}91.2 \pm \\
16.4\end{array}$ & $\begin{array}{l}86.8 \pm \\
16.1^{*}\end{array}$ & $\begin{array}{c}83.5 \pm \\
12.4^{*}\end{array}$ & 0.01 & $\begin{array}{c}90.9 \pm \\
19.6\end{array}$ & $\begin{array}{c}89.6 \pm \\
10.1^{*}\end{array}$ & $\begin{array}{c}86.1 \pm \\
10.2^{*}\end{array}$ & 0.02 & $\begin{array}{c}91.8 \pm \\
15.3\end{array}$ & $\begin{array}{c}87.7 \pm \\
10.3^{*}\end{array}$ & $\begin{array}{c}86.3 \pm \\
9.1^{*}\end{array}$ & 0.01 \\
\hline Fat mass (kg) & $\begin{array}{c}35.7 \pm \\
7.0\end{array}$ & $\begin{array}{c}32.5 \pm \\
7.1^{*}\end{array}$ & $\begin{array}{c}30.5 \pm \\
8.2^{*}\end{array}$ & 0.01 & $\begin{array}{c}36.9 \pm \\
11.1\end{array}$ & $\begin{array}{l}33.8 \pm \\
10.1^{*}\end{array}$ & $\begin{array}{c}31.8 \pm \\
10.0^{*}\end{array}$ & 0.02 & $\begin{array}{c}36.6 \pm \\
6.4\end{array}$ & $\begin{array}{c}33.1 \pm \\
8.1^{*}\end{array}$ & $\begin{array}{c}31.9 \pm \\
9.2^{*}\end{array}$ & 0.01 & $\begin{array}{c}37.1 \pm \\
9.1\end{array}$ & $\begin{array}{c}34.2 \pm \\
10.1^{*}\end{array}$ & $\begin{array}{c}33.9 \pm \\
10.0^{*}\end{array}$ & 0.04 \\
\hline WC (cm) & $\begin{array}{c}111.4 \pm \\
11.6\end{array}$ & $\begin{array}{c}107.9 \pm \\
11.7^{*}\end{array}$ & $\begin{array}{c}105.1 \pm \\
9.2^{*}\end{array}$ & 0.01 & $\begin{array}{c}111.4 \pm \\
9.2\end{array}$ & $\begin{array}{c}106.4 \pm \\
9.7^{*}\end{array}$ & $\begin{array}{c}104.2 \pm \\
10.1^{*}\end{array}$ & 0.03 & $\begin{array}{c}110.8 \pm \\
10.8\end{array}$ & $\begin{array}{c}106.8 \pm \\
9.1^{*}\end{array}$ & $\begin{array}{c}105.6 \pm \\
11.3^{*}\end{array}$ & 0.03 & $\begin{array}{c}106.3 \pm \\
10.9\end{array}$ & $\begin{array}{c}106.8 \pm \\
10.0^{*}\end{array}$ & $\begin{array}{c}105.3 \pm \\
8.1^{*}\end{array}$ & 0.04 \\
\hline WHR & $\begin{array}{c}0.96 \pm \\
0.07\end{array}$ & $\begin{array}{c}0.94 \pm \\
0.06\end{array}$ & $\begin{array}{c}0.94 \pm \\
0.1\end{array}$ & 0.34 & $\begin{array}{c}0.94 \pm \\
0.01\end{array}$ & $\begin{array}{c}0.93 \pm \\
0.1\end{array}$ & $\begin{array}{c}0.94 \pm \\
0.03\end{array}$ & 0.49 & $\begin{array}{c}0.95 \pm \\
0.07\end{array}$ & $\begin{array}{c}0.94 \pm \\
0.06\end{array}$ & $\begin{array}{c}0.94 \pm \\
0.08\end{array}$ & 0.67 & $\begin{array}{c}0.95 \pm \\
0.1\end{array}$ & $\begin{array}{c}0.94 \pm \\
0.09\end{array}$ & $\begin{array}{c}0.92 \pm \\
0.10\end{array}$ & 0.57 \\
\hline$S B(m m H g)$ & $\begin{array}{c}126.0 \pm \\
11.8\end{array}$ & $\begin{array}{c}122.7 \pm \\
13.7^{*}\end{array}$ & $\begin{array}{c}122.2 \pm \\
10.9^{*}\end{array}$ & 0.01 & $\begin{array}{c}128.7 \pm \\
12.3\end{array}$ & $\begin{array}{c}126.4 \pm \\
13.1^{*}\end{array}$ & $\begin{array}{c}125.9 \pm \\
11.2^{*}\end{array}$ & 0.03 & $\begin{array}{c}126.1 \pm \\
10.1\end{array}$ & $\begin{array}{c}122.3^{ \pm} \\
10.1^{*}\end{array}$ & $\begin{array}{c}122.8 \pm \\
12.8^{*}\end{array}$ & 0.03 & $\begin{array}{c}131.2 \pm \\
10.8\end{array}$ & $\begin{array}{c}126.5 \pm \\
12.1^{*}\end{array}$ & $\begin{array}{c}125.0 \pm \\
9.0^{*}\end{array}$ & 0.02 \\
\hline $\mathrm{DB}(\mathrm{mmHg})$ & $\begin{array}{c}81.1 \pm \\
10.5\end{array}$ & $\begin{array}{c}78.2 \pm \\
8.1\end{array}$ & $\begin{array}{c}78.1 \pm \\
12.0\end{array}$ & 0.48 & $\begin{array}{c}82.3 \pm \\
9.1\end{array}$ & $\begin{array}{c}80.1 \pm \\
8.1\end{array}$ & $\begin{array}{c}78.8 \pm \\
9.1\end{array}$ & 0.53 & $\begin{array}{c}80.4 \pm \\
10.2\end{array}$ & $75.9 \pm 6.4$ & $\begin{array}{c}78.9 \pm \\
6.0\end{array}$ & 0.24 & $\begin{array}{c}80.7 \pm \\
5.2\end{array}$ & $79.8 \pm 8.1$ & $\begin{array}{c}79.2 \pm \\
7.0\end{array}$ & 0.45 \\
\hline
\end{tabular}

H: High Protein/Low Carbohydrate; S: Standard; DB: Diastolic Blood Pressure; Mths: Months; SB: Systolic Blood Pressure; WHR: Waist to Hip Ratio; WC: Waist Circumference; $\left({ }^{*}\right)<0.05$, in each genotype group with basal values. No statistical differences between genotypes with Lys/lys vs Lys/Asn+Asn/Asn carriers in each diet. Quantitative variables with normal distribution were analyzed with a two-tailed, aired Student's-t test (weight, fat mass, BMI). Non-parametric parameters were analyzed with the Wilcoxon test (WC, WHR, SB, DB)

Table 1: Changes in Anthropometric Variables (Mean \pm S.D).

\begin{tabular}{|c|c|c|c|c|c|c|c|c|c|c|c|c|c|c|c|c|}
\hline \multirow[t]{3}{*}{ Characteristics } & \multicolumn{8}{|c|}{ DIET H (n=139) } & \multicolumn{8}{|c|}{ DIET S (n=141) } \\
\hline & \multicolumn{4}{|c|}{ LysLys(n=94) } & \multicolumn{4}{|c|}{ LysAsn+ AsnAsn ( $n=45)$} & \multicolumn{4}{|c|}{ LysLys ( $n=94)$} & \multicolumn{4}{|c|}{ LysAsn+ AsnAsn ( $n=47)$} \\
\hline & 0 time & $\begin{array}{c}\text { At } 3 \\
\text { months }\end{array}$ & $\begin{array}{c}\text { At } 9 \\
\text { months }\end{array}$ & $\begin{array}{c}p \\
\text { value }\end{array}$ & 0 time & $\begin{array}{c}\text { At } 3 \\
\text { months }\end{array}$ & $\begin{array}{c}\text { At } 9 \\
\text { months }\end{array}$ & $\begin{array}{c}\mathbf{p} \\
\text { value }\end{array}$ & 0 time & $\begin{array}{c}\text { At } 3 \\
\text { months }\end{array}$ & $\begin{array}{c}\text { At } 9 \\
\text { months }\end{array}$ & & 0 time & $\begin{array}{c}\text { At } 3 \\
\text { months }\end{array}$ & $\begin{array}{c}\text { At } 9 \\
\text { months }\end{array}$ & p value \\
\hline Glucose (mg/dl) & $\begin{array}{c}103.2 \pm \\
10.2\end{array}$ & $\begin{array}{c}99.1 \pm \\
13.1^{*}\end{array}$ & $\begin{array}{c}98.1 \pm \\
10.1^{*}\end{array}$ & 0.01 & $\begin{array}{c}100.9 \pm \\
12.2\end{array}$ & $\begin{array}{c}99.9 \pm \\
13.0\end{array}$ & $\begin{array}{l}98.5 \pm \\
9.8\end{array}$ & 0.22 & \begin{tabular}{|c|}
$101.4 \pm$ \\
11.1
\end{tabular} & $\begin{array}{c}98.7 \pm \\
9.2\end{array}$ & $\begin{array}{c}99.5 \pm \\
9.1\end{array}$ & 0.34 & $\begin{array}{c}98.9 \pm \\
9.4\end{array}$ & $96.8 \pm 6.3$ & $\begin{array}{c}98.3 \pm \\
7.1\end{array}$ & 0.45 \\
\hline $\begin{array}{l}\text { Total ch. (mg/ } \\
\text { dl) }\end{array}$ & $\begin{array}{c}209.1 \pm \\
19.8\end{array}$ & $\begin{array}{c}197.8 \pm \\
21.1^{\star}\end{array}$ & $\begin{array}{c}198.5 \pm \\
30.7^{*}\end{array}$ & 0.02 & $\begin{array}{c}206.6 \pm \\
21.1\end{array}$ & $\begin{array}{c}200.7 \pm \\
31.0\end{array}$ & $\begin{array}{c}207.4 \pm \\
10.2\end{array}$ & 0.38 & $\begin{array}{c}211.8 \pm \\
40.9\end{array}$ & $\begin{array}{c}199.0 \pm \\
30.4^{*}\end{array}$ & $\begin{array}{c}198.3 \pm \\
18.4^{*}\end{array}$ & 0.03 & $\begin{array}{c}203.6 \pm \\
21.2\end{array}$ & $\begin{array}{c}196.4 \pm \\
19.1\end{array}$ & $\begin{array}{c}193.8 \pm \\
12.9\end{array}$ & 0.34 \\
\hline LDL-ch. (mg/dl) & $\begin{array}{c}127.9 \pm \\
20.1\end{array}$ & $\begin{array}{c}119.7 \pm \\
20.9^{*}\end{array}$ & $\begin{array}{c}120.2 \pm \\
13.1^{*}\end{array}$ & 0.01 & $\begin{array}{c}126.9 \pm \\
27.2\end{array}$ & $\begin{array}{c}123.4 \pm \\
21.1\end{array}$ & $\begin{array}{c}129.1 \pm \\
12.1\end{array}$ & 0.41 & \begin{tabular}{|c|}
$125.2 \pm$ \\
18.5
\end{tabular} & $\begin{array}{c}118.1 \pm \\
23.2^{*}\end{array}$ & $\begin{array}{c}117.8 \pm \\
18.0^{*}\end{array}$ & 0.01 & $\begin{array}{c}124.6 \pm \\
18.1\end{array}$ & $\begin{array}{c}120.2 \pm \\
20.9\end{array}$ & $\begin{array}{c}119.9 \pm \\
19.8\end{array}$ & 0.09 \\
\hline HDL-ch. (mg/dl) & $\begin{array}{c}54.5 \pm \\
10.5\end{array}$ & $\begin{array}{c}53.7 \pm \\
8.1\end{array}$ & $\begin{array}{c}55.1 \\
7.1\end{array}$ & 0.78 & $\begin{array}{c}56.8 \pm \\
10.0\end{array}$ & $\begin{array}{c}56.3 \pm \\
9.0\end{array}$ & $\begin{array}{c}55.8 \pm \\
8.1\end{array}$ & 0.68 & $\begin{array}{c}56.2 \pm \\
11.2\end{array}$ & $\begin{array}{c}53.2 \pm \\
9.4\end{array}$ & $\begin{array}{c}52.7 \pm \\
11.0\end{array}$ & 0.55 & $\begin{array}{c}55.0 \pm \\
11.1\end{array}$ & $53.8 \pm 8.1$ & $\begin{array}{c}55.1 \pm \\
11.2\end{array}$ & 0.23 \\
\hline TG (mg/dl) & $\begin{array}{c}125.8 \\
43.1\end{array} \pm$ & $\begin{array}{c}114.1 \pm \\
30.4^{\star}\end{array}$ & $\begin{array}{c}106.1 \pm \\
22.2^{*}\end{array}$ & 0.01 & $\begin{array}{c}120.3 \pm \\
20.1\end{array}$ & $\begin{array}{c}112.8 \pm \\
33.2^{*}\end{array}$ & $\begin{array}{c}107.9 \pm \\
20.3^{\star}\end{array}$ & 0.03 & $\begin{array}{c}130.1 \pm \\
42.6\end{array}$ & $\begin{array}{c}119.4 \pm \\
33.1^{*}\end{array}$ & $\begin{array}{c}118.1 \pm \\
30.1^{*}\end{array}$ & 0.01 & $\begin{array}{c}121.0 \pm \\
40.3\end{array}$ & $\begin{array}{c}119.9 \pm \\
18.3\end{array}$ & $\begin{array}{c}117.9 \pm \\
30.9\end{array}$ & 0.56 \\
\hline Insulin (mUI/L) & $\begin{array}{c}10.8 \pm \\
5.4\end{array}$ & $\begin{array}{c}9.0 \pm \\
5.2^{*}\end{array}$ & $\begin{array}{c}8.2 \pm \\
5.0^{\star}\end{array}$ & 0.03 & $\begin{array}{c}11.6 \pm \\
7.1\end{array}$ & $\begin{array}{c}10.4 \pm \\
9.1\end{array}$ & $\begin{array}{c}9.8 \pm \\
7.4\end{array}$ & 0.18 & $\begin{array}{c}10.9 \pm \\
5.0\end{array}$ & $9.1 \pm 4.9$ & $9.4 \pm 4.2$ & 0.09 & $\begin{array}{c}10.7 \pm \\
5.0\end{array}$ & $10.5 \pm 4.2$ & $8.9 \pm 7.0$ & 0.45 \\
\hline HOMA & $\begin{array}{c}2.2 \pm \\
0.9\end{array}$ & $\begin{array}{c}2.0 \pm \\
0.8^{\star}\end{array}$ & $\begin{array}{c}1.7 \pm \\
1.1^{*}\end{array}$ & 0.02 & $\begin{array}{c}2.6 \pm \\
1.1\end{array}$ & $2.7 \pm 2.1$ & $\begin{array}{c}1.9 \pm \\
1.3\end{array}$ & 0.09 & $2.3 \pm 1.1$ & $2.0 \pm 1.0$ & $2.1 \pm 1.1$ & 0.28 & $2.3 \pm 1.2$ & $2.2 \pm 2.0$ & $2.4 \pm 1.6$ & 0.34 \\
\hline CR (mg/dl) & $\begin{array}{c}4.8 \pm \\
3.1\end{array}$ & $\begin{array}{l}4.7 \pm \\
3.2\end{array}$ & $\begin{array}{l}2.6 \pm \\
3.0\end{array}$ & 0.03 & $\begin{array}{c}4.4 \pm \\
3.1\end{array}$ & $5.1 \pm 4.0$ & $\begin{array}{c}3.9 \pm \\
3.2\end{array}$ & 0.19 & $5.0 \pm 4.0$ & $5.3 \pm 4.1$ & $4.9 \pm 4.0$ & 0.16 & $5.2 \pm 4.0$ & $5.1 \pm 3.9$ & $5.3 \pm 3.1$ & 0.45 \\
\hline
\end{tabular}

H: High Protein/Low Carbohydrate; S: Standard; Chol: Cholesterol; TG: Triglycerides; CR: C Reactive Protein; HOMA: Homeostasis Model Assessment; Mths: Months (*) $<0.05$, in each group with basal values. No statistical differences among genotypes in each diet or in different diet groups. Quantitative variables with normal distribution were analyzed with a two-tailed, aired Student's-t test (glucose, Insulin, HOMA-IR, HDL cholesterol, C reactive protein). Non-parametric parameters were analyzed with the Wilcoxon test (total cholesterol, LDL cholesterol, tryglicerides). 
Citation: de Luis D, Aller R, Izaola O, Primo D, Romero E (2016) Effects of a High Protein/Low Carbohydrate Versus a Standard Hypocaloric Diet on Weight and Cardiovascular Risk Factors, Role of a Lys656asn Polymorphism of Leptin Receptor Gene. J Metabolic Synd 5: 206. doi:10.4172/2167-0943.1000206

Page 4 of 5

\begin{tabular}{|c|c|c|c|c|c|c|c|c|c|c|c|c|c|c|c|c|}
\hline \multirow[t]{3}{*}{ Characteristics } & \multicolumn{8}{|c|}{ DIET H ( $n=139)$} & \multicolumn{8}{|c|}{ DIET S (n=141) } \\
\hline & \multicolumn{4}{|c|}{ LysLys(n=94) } & \multicolumn{4}{|c|}{ LysAsn+ AsnAsn $(n=45)$} & \multicolumn{4}{|c|}{ LysLys (n=94) } & \multicolumn{4}{|c|}{ LysAsn+ AsnAsn (n=47) } \\
\hline & 0 time & $\begin{array}{c}\text { At } 3 \\
\text { months }\end{array}$ & $\begin{array}{c}\text { At } 9 \\
\text { months }\end{array}$ & $p$ value & 0 time & $\begin{array}{c}\text { At } 3 \\
\text { months }\end{array}$ & $\begin{array}{c}\text { At } 9 \\
\text { months }\end{array}$ & $p$ value & 0 time & $\begin{array}{c}\text { At } 3 \\
\text { months }\end{array}$ & $\begin{array}{c}\text { At } 9 \\
\text { months }\end{array}$ & $p$ value & 0 time & $\begin{array}{c}\text { At } 3 \\
\text { months }\end{array}$ & $\begin{array}{c}\text { At } 9 \\
\text { months }\end{array}$ & $p$ value \\
\hline $\begin{array}{c}\text { Adionectin (ng/ } \\
\mathrm{ml})\end{array}$ & $\begin{array}{c}10.2 \pm \\
6.0\end{array}$ & $\begin{array}{c}9.6 \pm \\
5.1\end{array}$ & $\begin{array}{c}10.3 \pm \\
8.0\end{array}$ & 0.23 & $\begin{array}{c}10.8 \pm \\
7.0\end{array}$ & $\begin{array}{l}11.1 \pm \\
9.0\end{array}$ & $\begin{array}{c}10.9 \pm \\
7.2\end{array}$ & 0.34 & $\begin{array}{c}11.0 \pm \\
4.1\end{array}$ & $\begin{array}{c}10.8 \pm \\
4.0\end{array}$ & $\begin{array}{l}9.9 \pm \\
4.3\end{array}$ & 0.34 & $\begin{array}{c}9.9 \pm \\
5.1\end{array}$ & $\begin{array}{c}10.6 \pm \\
4.9\end{array}$ & $\begin{array}{c}10.8 \pm \\
4.1\end{array}$ & 0.43 \\
\hline $\begin{array}{l}\text { Resistin (ng/ } \\
\text { ml) }\end{array}$ & $\begin{array}{l}7.4 \pm \\
3.2\end{array}$ & $\begin{array}{l}7.2 \pm \\
5.0\end{array}$ & $\begin{array}{l}6.9 \pm \\
4.3\end{array}$ & 0.45 & $\begin{array}{l}7.3 \pm \\
3.1\end{array}$ & $\begin{array}{l}7.0 \pm \\
3.2\end{array}$ & $\begin{array}{l}7.1 \pm \\
3.1\end{array}$ & 0.45 & $\begin{array}{l}8.0 \pm \\
2.5\end{array}$ & $\begin{array}{l}7.9 \pm \\
3.1\end{array}$ & $\begin{array}{l}7.7 \pm \\
2.8\end{array}$ & 0.45 & $\begin{array}{l}8.0 \pm \\
3.1\end{array}$ & $\begin{array}{l}7.1 \pm \\
4.0\end{array}$ & $\begin{array}{l}6.9 \pm \\
4.0\end{array}$ & 0.31 \\
\hline Letin (ng/ml) & $\begin{array}{c}31.8 \pm \\
17.4\end{array}$ & $\begin{array}{l}21.9 \pm \\
13.1^{*}\end{array}$ & $\begin{array}{l}15.3 \pm \\
10.1^{*}\end{array}$ & 0.01 & $\begin{array}{c}39.1 \pm \\
10.9\end{array}$ & $\begin{array}{l}20.8 \pm \\
9.2^{*}\end{array}$ & $\begin{array}{c}18.5 \pm \\
9.5^{*}\end{array}$ & 0.01 & $\begin{array}{c}31.9 \pm \\
13.0\end{array}$ & $\begin{array}{l}20.3 \pm \\
8.0^{*}\end{array}$ & $\begin{array}{l}15.0 \pm \\
6.1^{*}\end{array}$ & 0.01 & $\begin{array}{c}41.9 \pm \\
10.1\end{array}$ & $\begin{array}{l}22.7 \pm \\
9.3^{*}\end{array}$ & $\begin{array}{c}21.6 \pm \\
8.1^{*}\end{array}$ & 0.02 \\
\hline
\end{tabular}

$\left(^{*}\right)<0.05$, in each group with basal values. No statistical differences among genotypes in each diet or in different diet groups. Quantitative variables with normal distribution were analyzed with a two-tailed, aired Student's-t test (resistin). Non-parametric parameters were analyzed with the Wilcoxon test (leptin and adiponectin).

Table 3: Circulating Adyocitokines (mean \pm S.D).

unchanged in patients with Asn allele carriers. With the diet $S$ and in patients with Lys656Asn genotypes, only total cholesterol $(-13.2 \pm$ $7.1 \mathrm{mg} / \mathrm{dl}$ vs $-5.1 \pm 8.2 \mathrm{mg} / \mathrm{dl} ; \mathrm{p}<0.05)$, triglycerides $(-12.1 \pm 7.1 \mathrm{mg} /$ $\mathrm{dl}$ vs $-4.1 \pm 7.91 \mathrm{mg} / \mathrm{dl} ; \mathrm{p}<0.05)$ and LDL- total cholesterol $(-5.7 \pm 3.1$ $\mathrm{mg} / \mathrm{dl}$ vs $-2.1 \pm 5.1 \mathrm{mg} / \mathrm{dl} ; \mathrm{p}<0.05)$ decreased. Lipid profile remained unchanged in patients with Asn allele genotype.

Table 3 reports levels of adipokines. With the diet HP and in both group of genotypes, leptin levels $(-16.3 \pm 12.1 \mathrm{ng} / \mathrm{ml}$ vs. $-20.0 \pm 11.1 \mathrm{ng} /$ $\mathrm{ml}: \mathrm{ns})$ decreased. With the diet $\mathrm{S}$, leptin levels $(-16.9 \pm 5.2 \mathrm{ng} / \mathrm{ml} \mathrm{vs}$. $-21.1 \pm 4.3 \mathrm{ng} / \mathrm{ml}$ : ns) decreased in both groups, too. The decrease of leptin levels was similar with diet HP and S.

\section{Discussion}

Findings from our study suggest that, in obese patients following a high protein hypocaloric diet, carriers of the Asn 656 allele of the LEPR gene do not respond with a decrease of LDL cholesterol, insulin levels and HOMA-IR after a significant weight loss. These obese patients (Asn carriers) do not show a decrease of triglyceride and LDL cholesterol levels after a significant weight loss with a normal hypocaloric diet. After 9 months of two type of hypocaloric diets significantly reduced BMI, weight, fat mass, waist circumference and systolic blood pressure in obese subjects with different genotypes.

Several population-based studies have been realized to study the effects of this SNP in the leptin receptor gene (LEPR) on BMI. A meta-analysis of linkage and association of LEPR SNPs with BMI reported that, although certain genotypic effects could be populationspecific, there was no evidence that any allele (Lys109Arg, Gln223Arg, Lys656Asn) is associated with body mass index or waist circumference [14]. Our results (basal and post treatment) are in agreement with the literature.

Interventional designs with this SNP are limited. Rossum et al. [15] did not show relation between Lys656Asn SNP and weight regain in a cohort in The Netherlands. Other study [5] demonstrated the different changes in weight loss and leptin response secondary to a hypocaloric diet with a $25 \%$ of calories from fats and with $<20 \%$ of monounsaturated fats in relation with Lys656Asn SNP. In other design of our group, the weight loss secondary to a high polyunsaturated fatty acid (PUFA) diet produced, in carriers of ASn656 allele, a different change than nonAsn allele carriers, with a lack of improvement in insulin, leptin levels, and HOMA-IR. Therefore, obese subjects with this mutant allele have a better lipid profile after weight loss [7]. This better metabolic response in obese subjects without Asn allele has been detected with a high monounsaturated fatty acid (PUFA) diet, too [16]. In a randomized clinical trial [6] with 2 different hypocaloric diets (a low fat versus a low carbohydrate diet), a different metabolic response was observed, too.
Only leptin levels have a significant decrease in subjects with genotype Lys656Lys secondary to weight loss with both diets. The changes in serum leptin concentration due to 2 months' intervention with low fat are higher than with a low carbohydrate diet (low fat diet: $30.3 \%$; $<0.05$ ) and (low carbohydrate diet: $15.5 \%$; $<<0.05$ ).

A limitation of previous studies is the short term intervention (2-3 months), in our present study dietary intervention has been realized during 9 months. The longest intervention study in the literature was one year after the realization of a biliopancreatic derivation [17]. In both genetic groups, BMI, weight, glucose, triglyceride, total cholesterol, LDL cholesterol, and blood pressure decreased. Initial weight percent loss at 1 year of follow-up was higher in mutant group than in wildtype group $(38.9 \%$ vs $29.9 \%$; $<0.05)$. In our present study, after a high protein hypocaloric diet, subjects with Lys656Lys genotype of the LEPR gene showed a decrease of LDL cholesterol, insulin levels and HOMA-IR after weight loss. These obese subjects showed a decrease of LDL cholesterol and triglyceride levels after standard hypocaloric diet. 9 months of both diets significantly reduced weight, body mass index, waist circumference, fat mass and systolic blood pressure in obese subjects with both genotypes, with poorer biochemical response in patients carrying the allele Asn. The decrease of leptin levels was similar in both diets and in both genotypes.

Other authors have obtained association between SNP on codon 656 and obesity parameters. In postmenopausal females with intolerance of glucose (ITG), relationships were found with Lys656Asn for fasting insulin, as well as in response to an oral glucose test. In premenopausal females with ITG, associations were detected with Lys656Asn for overall glucose response to the glucose load [18]. In other studies, differences in substrate oxidation rates were found. In fasting conditions, Lys656Lys showed a trend to oxidize more carbohydrates and less fat than Asn656 carriers [19].

These different metabolic responses could be related with inclusion criteria and heterogeneity of subjects in the intereventional studies, distribution of nutrients of the hypocaloric diets, time course of dietary intervention and interaction with other polymorphisms in LEPR gene. For example Xenachis et al. [20] have study the response to a hypocaloric diet in the LEPR gene polymorphism, but in a different polymorphisms (Thr343Ser). The overweight women carried the Ser allele lost more weight in response to low calorie diet than non carriers. At last, other theories could be explained through an association of genetic variation in this receptor with the functionality of Leptin receptor and, therefore, influenced the leptin response and weight lost to energy restriction. Mars et al. [21] had observed heterogeneity of metabolic responses with the Lys109Arg, Gln223Arg and Lys656Asn SNPS in the initial decline in leptin after a hypocaloric diet with a $21.5 \%$ of fats. 
Citation: de Luis D, Aller R, Izaola O, Primo D, Romero E (2016) Effects of a High Protein/Low Carbohydrate Versus a Standard Hypocaloric Diet on Weight and Cardiovascular Risk Factors, Role of a Lys656asn Polymorphism of Leptin Receptor Gene. J Metabolic Synd 5: 206. doi:10.4172/2167-0943.1000206

Page 5 of 5

In conclusion, carries of ASn656 allele have a different metabolic change than wild genotype obese, with a lack of decrease of HOMAIR, insulin levels, total cholesterol and LDL cholesterol after a high protein hypocaloric diet and a lack of decrease of HOMA-IR, insulin, total cholesterol, LDL cholesterol and triglycerides after a standard hypocaloric diet. This polymorphism did not influence in terms of weight loss.

\section{Authors Contribution}

Daniel Antonio de Luis designed the study an wrote the article. R Aller realized nutritional evaluation. O IZaola realized nutritional evaluation. D Primo realized laboratory analysis. E Romero designed the study an wrote the article.

\section{References}

1. Meier U, Gressner AM (2004) Endocrine regulation of energy metabolism: review of pathobiochemical and clinical chemical aspects of leptin, ghrelin adiponectin and resistin. Clin Chem 50: 1511-1525.

2. de Luis DA, Castrillon JLP, Dueñas A (2009) Leptin and Obesity. Minerva Med 100: 229-236.

3. Considine RV, Sinha MK, Heiman ML, Kriauciunas A, Stephens TW, et al (1996) Serum immunorective-leptin concentrations in normal-weight and obese humans. N engl J Med 334: 292-295.

4. Heo M, Leíble RL, Boyer BB, Cheng WK, Koulu M, et al. (2001) Pooling analisis of genetic data: The assocation of leptin receptor (LEPR) polymorphisms with variables related to human adiposity. Genetics 159: 1163-1178.

5. de Luis Roman D, de la Fuente RA, Sagrado MG, Izaola O, Vicente RC (2006) Leptin receptor Lys656Asn polymorphism is associated with decreased leptin response and weight loss secondary to a lifestyle modification in obese patients. Arch Med Res 37: 854-859.

6. de Luis DA, Aller R, Izaola O, Sagrado MG, Conde R (2008) Influence Of Lys656Asn polymorphism of leptin receptor gene on leptin response secondary to two hypocaloric diets: a randomized clinical trial. Ann Nutr Metab 52: 209214.

7. de Luis DA, Aller R, Izaola O, Sagrado MG, Conde R, et al. (2015) Effect of Lys656Asn Polymorphism of Leptin Receptor Gene on Cardiovascular Risk Factors and Serum Adipokine Levels after a High Polyunsaturated Fat Diet in Obese Patients. J Clin Lab Anal 29: 432-436.

8. de Luis DA, Aller R, Izaola O, Sagrado MG, Conde R (2008) Influence of ALA54THR polymorphism of FABP-2 on weight loss and insulin levels secondary to two hypocaloric diets: a randomized clinical trial. Diabetes Res Clin Pract 82: 113-118.
9. Santos FL, Esteves SS, da Costa Pereira A, Yancy WS Jr, Nunes JP (2012) Systematic review and meta-analysis of clinical trials of the effects of low carbohydrate diets on cardiovascular risk factors. Obes Rev 13: 1048-1066.

10. Mataix J, Mañas M (1998) Tablas de composición de alimentos españoles. Ed: University of Granada.

11. Lukaski HC, Johnson PE, Bolonchuk WW, Lykken GI (1985) Assessment of fat-free mass using bioelectrical impedance measurements of the human body. Am J Clin Nutr 41: 810-817.

12. Mathews DR, Hosker JP, Rudenski AS, Naylor BA, Treacher DF, et al. (1985) Homesotasis model assessment: insulin resistance and beta cell function from fasting plasma glucose and insulin concentrations in man. Diabetologia 28 : 412-414.

13. Duart MJD, Arroyo CO, Frígols JLM (2002) Validation of a insulin model for the reactions in RIA. Clin Chem Lab Med 40: 1161-1167.

14. Heo M, Leibel RL, Fontaine KR, Boyer BB, Chung WK, et al. (2002) A metaanalytic investigation of linkage and association of common leptin receptor (LEPR) polymorphism with body mass index and waist circumference. Int $J$ Obes Relat Metab Disord 26: 640-646.

15. Rossum CTM, Hoebee B, van Baak M, Mars M, Saris WHM, et al. (2003) Genetic variation in the leptin receptor gene, leptin, and weight gain in young Dutch Adults. Obes Res 11: 377-386.

16. de Luis DA, Aller R, Izaola O, Conde R, Bouza JE (2013) Lys656Asn polymorphism of leptin receptor gene is related with leptin changes after a high monounsaturated fat diet in obese patients. J Investig Med 61: 286-290.

17. de Luis DA, Aller R, Sagrado MG, Izaola O, Terroba MC, et al. (2010) Influence of lys656asn polymorphism of leptin receptor gene on surgical results of biliopancreatic diversion. J Gastrointest Surg 14: 899-903.

18. Wauters M, Mertens I, Rankinen T, Chagnon C, Bouchard C, et al. (2001) Leptin receptor gene polymorphisms are associated with insulin in obese women with impaired glucose tolerance. J Clin Endocrinol Metab 86: 3227 3232.

19. Wauters M, Considine R, Chagnon M, Mertens I, Rankinen T, et al. (2002) Leptin levels, leptin receptor gene polymorphisms, and energy metabolism in women. Obes Res10: 394-400.

20. Xenachis C, Samojlik E, Raghuwanshi MP, Kirschner MA (2001) Leptin, insulin and TNF-alpha in weight loss. J Endocrinol Invest 24: 865-870.

21. Mars M, Van Rossum C, de Graaf C, Hoebee B, de Groot L, et al. (2004) Leptin responsiveness to energy restriction: Genetic variation in the leptin recepto gene. Obes Res 12: 442-446. 